\title{
Ammonia Gas Detection by Tannic Acid Functionalized and Reduced Graphene Oxide at Room Temperature
}

\author{
Sweejiang Yoo, Xin Li, Yuan Wu, Weihua Liu, Xiaoli Wang, and Wenhui Yi \\ School of Electronics and Information Engineering, Xian Jiaotong University, Xi'an, Shaanxi 710049, China \\ Correspondence should be addressed to Xin Li; 1x@mail.xjtu.edu.cn
}

Received 29 December 2013; Accepted 14 February 2014; Published 19 March 2014

Academic Editor: Zhenhui Kang

Copyright (C) 2014 Sweejiang Yoo et al. This is an open access article distributed under the Creative Commons Attribution License, which permits unrestricted use, distribution, and reproduction in any medium, provided the original work is properly cited.

\begin{abstract}
Reduced graphene oxide ( $\mathrm{rGO}$ ) based chemiresistor gas sensor has received much attention in gas sensing for high sensitivity, room temperature operation, and reversible. Here, for the first time, we present a promising chemiresistor for ammonia gas detection based on tannic acid (TA) functionalized and reduced graphene oxide $\left(\mathrm{rGO}_{\mathrm{TA}}\right.$ functionalized $)$. Green reductant of TA plays a major role in both reducing process and enhancing the gas sensing properties of $\mathrm{rGO}_{\mathrm{TA}}$ functionalized. Our results show $\mathrm{rGO}_{\mathrm{TA}}$ functionalized only selective to ammonia with excellent respond, recovery, respond time, and recovery times. $\mathrm{rGO}_{\mathrm{TA} \text { functionalized }}$ electrical resistance decreases upon exposure to $\mathrm{NH}_{3}$ where we postulated that it is due to $\mathrm{n}$-doping by TA and charge transfer between $\mathrm{rGO}_{\text {TA functionalized }}$ and $\mathrm{NH}_{3}$ through hydrogen bonding. Furthermore, $\mathrm{rGO}_{\mathrm{TA}}$ functionalized hinders the needs for stimulus for both recovery and respond. The combination of greener sensing material and simplicity in overall sensor design provides a new sight for green reductant approach of rGO based chemiresistor gas sensor.
\end{abstract}

\section{Introduction}

There has been a growing interest in detection of ammonia gas to serve the purpose of detection and monitoring for the field of environmental, automotive, chemical industry, and medical diagnostic [1]. Ammonia gas detection also contributes to explosive detection as ammonium nitrate based explosive releases a trace of ammonia gas when gradually decomposes [2]. On top of that, leakage detection and environment pollution monitoring are the primary concerns as the toxic and corrosive behaviours of ammonia possess severe threat to human skin, eyes, and respiratory tract. The conventional solid state sensor and conducting polymer sensor are suffering from low detection under room temperature. Besides, solid state sensor has the drawbacks of selectivity and complex fabrication process while conducting polymer sensor is often irreversible [3-7]. The emerging of carbon nanocomposites based chemiresistor especially reduced graphene oxide ( $\mathrm{rGO}$ ) has demonstrated high potential in gas sensing due to high sensitivity, room temperature operation, low power consumption, miniaturization potential, and reversibility, in which posing a trend of surpassing the conventional solid state and conducting polymer sensor. However, rGO chemiresistor is suffering from drawback of poor selectivity where functionalization and use of arrays are often the solution to improve selectivity $[8,9]$. Generally, environmental harmful reductant such as hydrazine or sodium borohydride are usually employed in synthesize of rGO. Thus, there is a demand in finding environment benign approach such as green, electrochemical, and solvothermal reductants [10-14].

Tannic acid (TA) is a water soluble phenolic hydroxylrich compound and is widely present in woods such as oak, walnut, and mahogany $[15,16]$. TA has demonstrated itself as an effective green reducing and stabilizing agent on synthesis of graphene, noble metal nanoparticles, and noble metal nanoparticles/graphene nanocomposites [17-20]. Recently, Zhang et al. have reported TA functionalized rGO with silver nanoparticles decorated for $\mathrm{H}_{2} \mathrm{O}_{2}$ and glucose sensing application [19]. However, TA functionalized rGO solely as gas sensor is rarely reported, in particular on ammonia gas detection. In this paper, for the first time, we demonstrate a simple yet green chemiresistor sensor for ammonia gas detection which comprises just overlapping comb electrode 


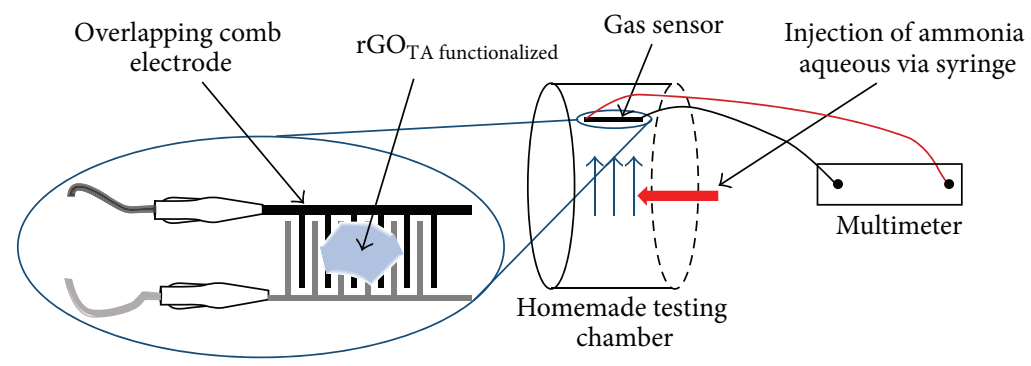

FIGURE 1: Schematic diagram of experiment set up for gas testing.

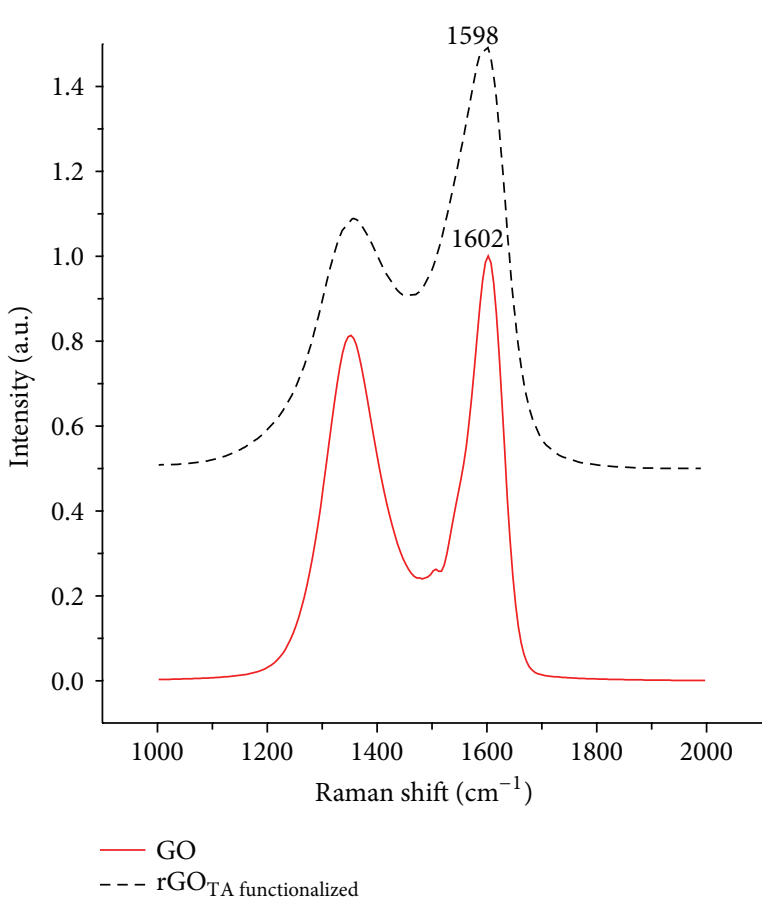

Figure 2: Raman spectra of $\mathrm{GO}$ and $\mathrm{rGO}_{\mathrm{TA}}$ functionalized. $I_{G}$ peak blue shifted from $1602 \mathrm{~nm}(\mathrm{GO})$ to $1598 \mathrm{~nm}$ ( $\mathrm{rGO}_{\mathrm{TA}}$ functionalized $) . I_{D} / I_{G}$ ratio is diminished from $0.81(\mathrm{GO})$ to $0.59\left(\mathrm{rGO}_{\mathrm{TA}}\right.$ functionalized $)$.

with drop-casted $\mathrm{rGO}_{\mathrm{TA}}$ functionalized. We employed the green reductant of TA to serve as both reducing and functionalizing agent for $\mathrm{rGO}_{\mathrm{TA}}$ functionalized, where TA plays a major role in reduction process and enhancing the gas sensing properties. Experimental findings indicate that $\mathrm{rGO}_{\mathrm{TA}}$ functionalized has fulfilled all the aspects to be considered as an excellent sensor for ammonia gas detection. In addition, $\mathrm{rGO}_{\mathrm{TA}}$ functionalized has the advantages of room temperature operation, has wide range detection, and hinders the need of stimulus such as current, purging of gas, and UV illumination for recovery/response.

\section{Experimental Section}

2.1. Preparation of $r G O_{T A}$ functionalized. Reduction of $\mathrm{GO}$ by TA was referring to a method reported by Lei et al. in 2011 with modification where reduction time was elongated to 24

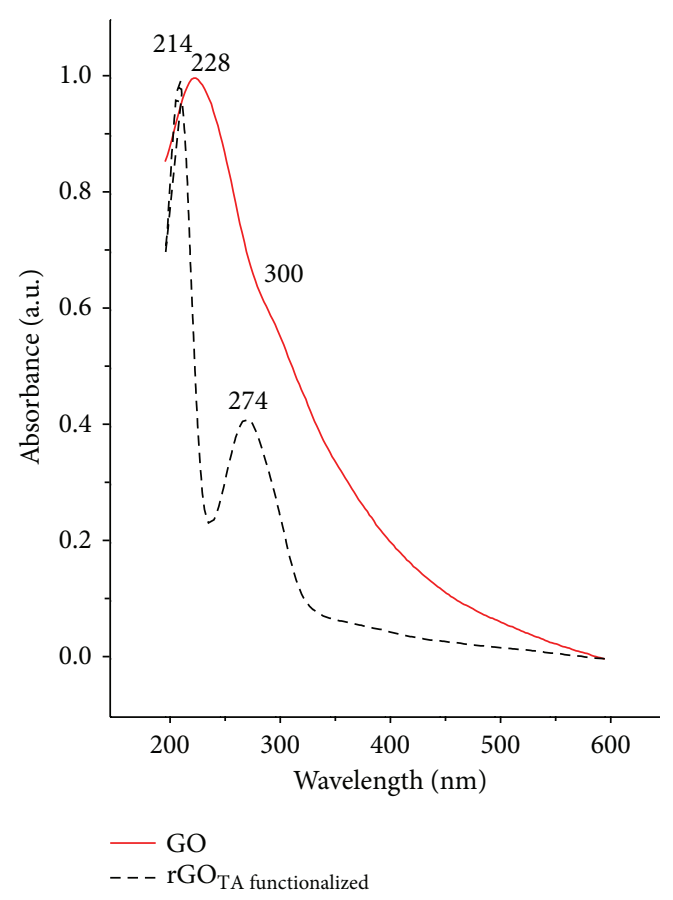

FIGURE 3: Absorption peak of $\mathrm{GO}$ and $\mathrm{rGO}_{\mathrm{TA}}$ functionalized. $\mathrm{GO}$ has absorption peaks at $228 \mathrm{~nm}$ and around $304 \mathrm{~nm}$ (small shoulder peak). TA has absorption peaks around 213 and $276 \mathrm{~nm}$ which are assigned for $\Pi-\Pi^{*}$ aromatic units and $\mathrm{C}=\mathrm{O}$ groups, respectively.

hours and $\mathrm{pH}$ was adjusted to 9 , with the aim to promote the degree of reduction and absorption of TA on rGO [21]. Graphene oxide (XF002) was obtained from XF Nano Inc., China. Tannic acid was obtained from Tianli Chemical Reagent Co. Ltd, China, without further purification. GO was dissolved in DI water and subjected to sonication. TA was dissolved in $90 \mathrm{~mL}$ of DI water and temperature was raised to $80^{\circ} \mathrm{C}$. Sodium hydroxide was added to adjust the $\mathrm{pH}$ to 9 . GO was then dropwise added into TA solution. The reaction mixture was kept continuous stirring and maintained at $80^{\circ} \mathrm{C}$ for 24 hours. The resultant suspension showed a black solution which indicated the reduction of GO to rGO. The resultant suspension was collected and washed three times. The sample was recorded as $\mathrm{rGO}_{\mathrm{TA}}$ functionalized. $\mathrm{rGO}_{\mathrm{TA}}$ functionalized was drop casted onto an array of electrode (overlapping comb electrode) and dried in oven. 


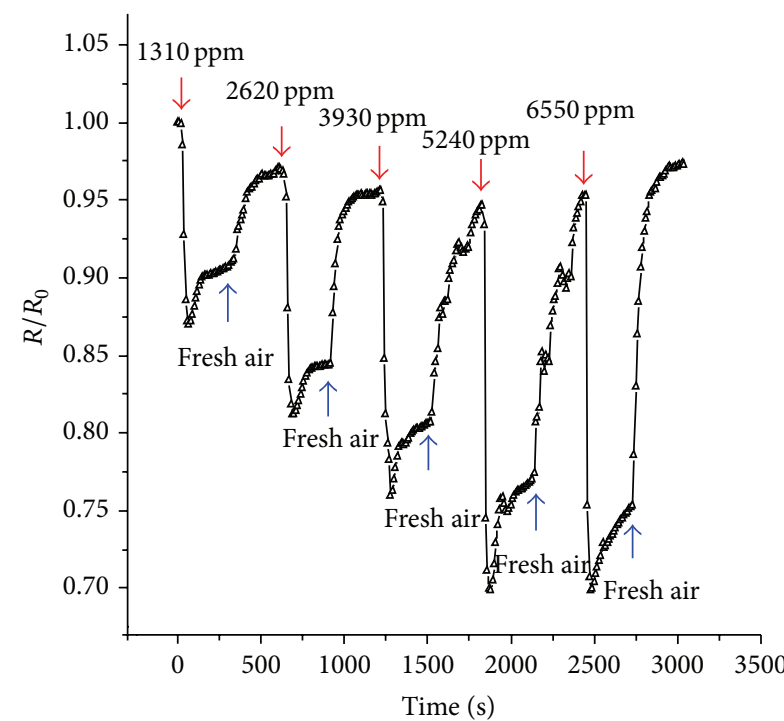

(a)

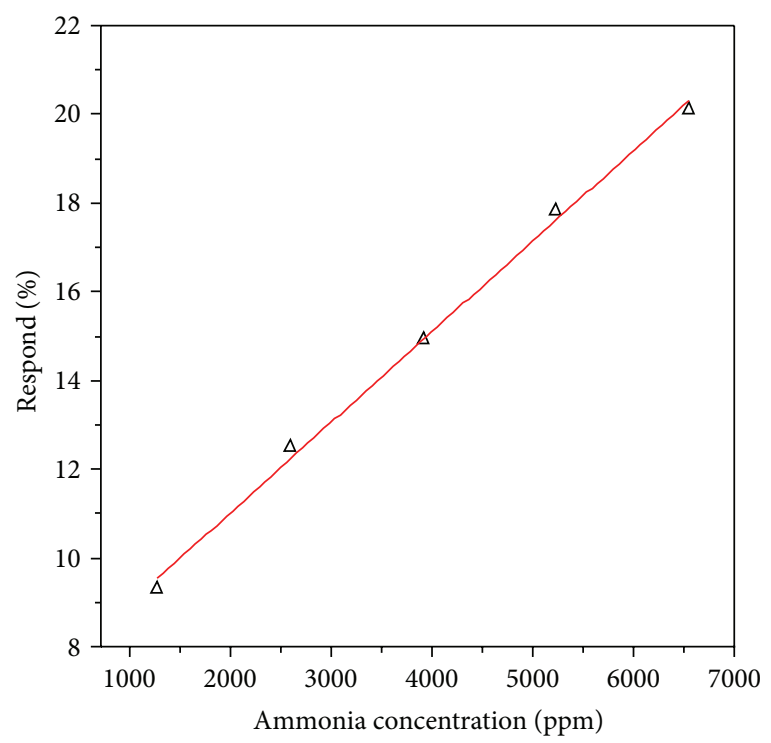

(c)

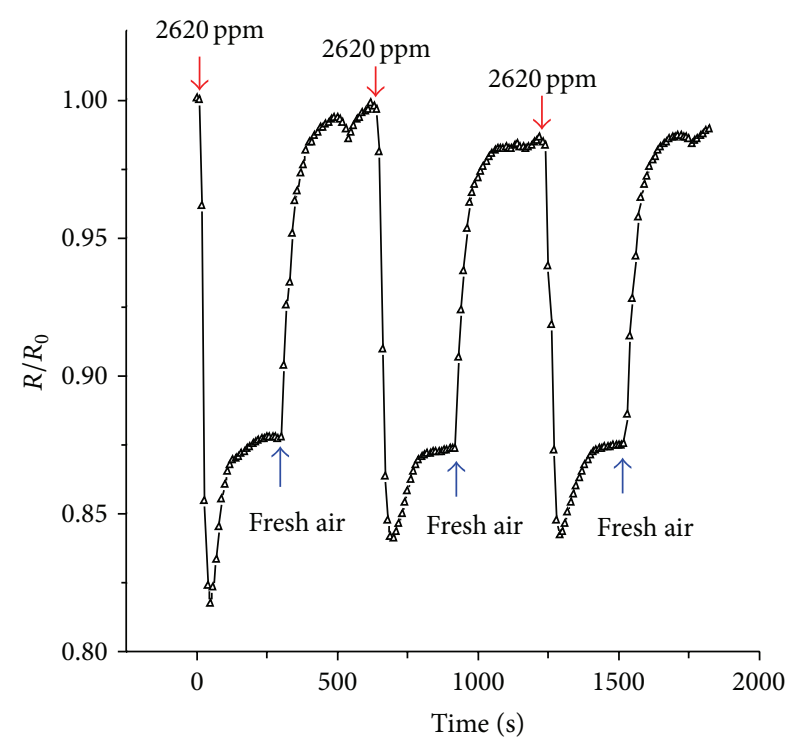

(b)

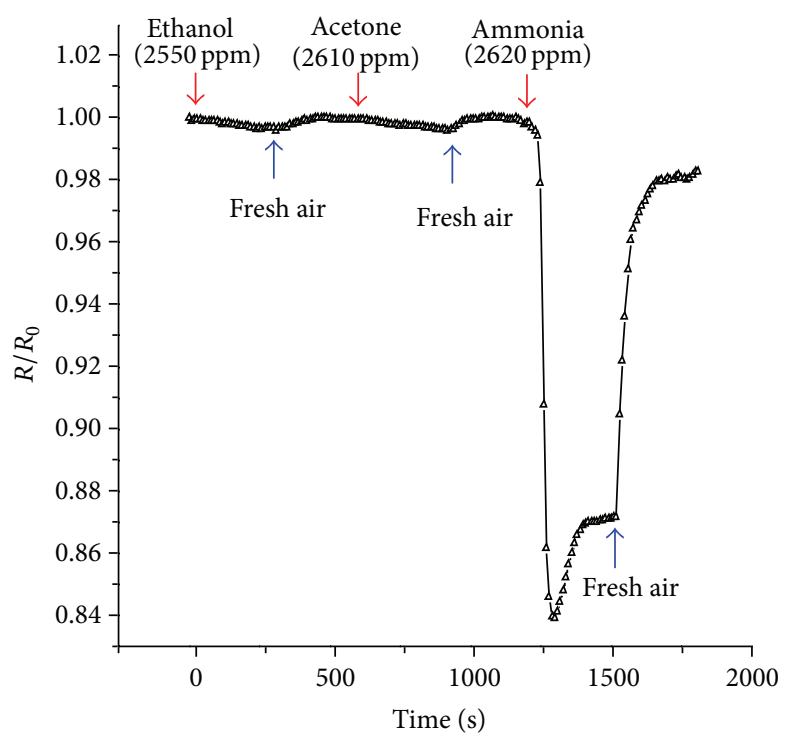

(d)

Figure 4: (a) Respond towards variation of $\mathrm{NH}_{3}$ concentration. (b) Respond when exposed to cycles of $\mathrm{NH}_{3}$. (c) The linear respond with increase of $\mathrm{NH}_{3}$ concentration. (d) Selectivity respond when exposed to ethanol, acetone, and ammonia.

2.2. Material Characterization and Gas Testing. All the UVvis absorption spectra were conducted on a Hitachi U3010 spectrophotometer. Raman spectra were conducted by Horiba Jobin Yvon HR 800 with $514 \mathrm{~nm}$ excitation. Raman spectra were fitted by Voigt function using peak fitting function of Origin 9.0.

Keithley Multimeter 2000 was used as electrical resistance measurement. Ammonia gas sensing experiments were carried out in a simple homemade testing chamber as shown in Figure 1 with a net volume of $5655 \mathrm{~cm}^{3}$. The ammonia aqueous was injected into chamber with a distance of $10 \mathrm{~cm}$ from sensor. Various concentrations of ammonia aqueous and organic solutions were introduced to the chamber manually via injection at the humidity of $26 \%$ and temperature of $25^{\circ} \mathrm{C}$. The door of chamber was opened to introduce fresh air for recovery process.

\section{Result and Discussion}

3.1. Synthesis and Characterization of $r G O_{T A}$ functionalized. From Raman spectra (Figure 2), $I_{G}$ peak is found blue shifted from $1602 \mathrm{~nm}(\mathrm{GO})$ to $1598 \mathrm{~nm}\left(\mathrm{rGO}_{\mathrm{TA}}\right.$ functionalized $)$ upon reduction. The blue shifting of $I_{G}$ peak indicated the recovery of hexagonal network upon reduction. The $I_{D} / I_{G}$ ratio which was often used to indicate the degree of disorder or defects in rGO is diminished from $0.81(\mathrm{GO})$ to $0.59\left(\mathrm{rGO}_{\mathrm{TA}}\right.$ functionalized $)$ which is suggesting the possible decrement in disorder due to reduction process. GO is reduced by TA via two-step $\mathrm{S}_{\mathrm{N}} 2$ 


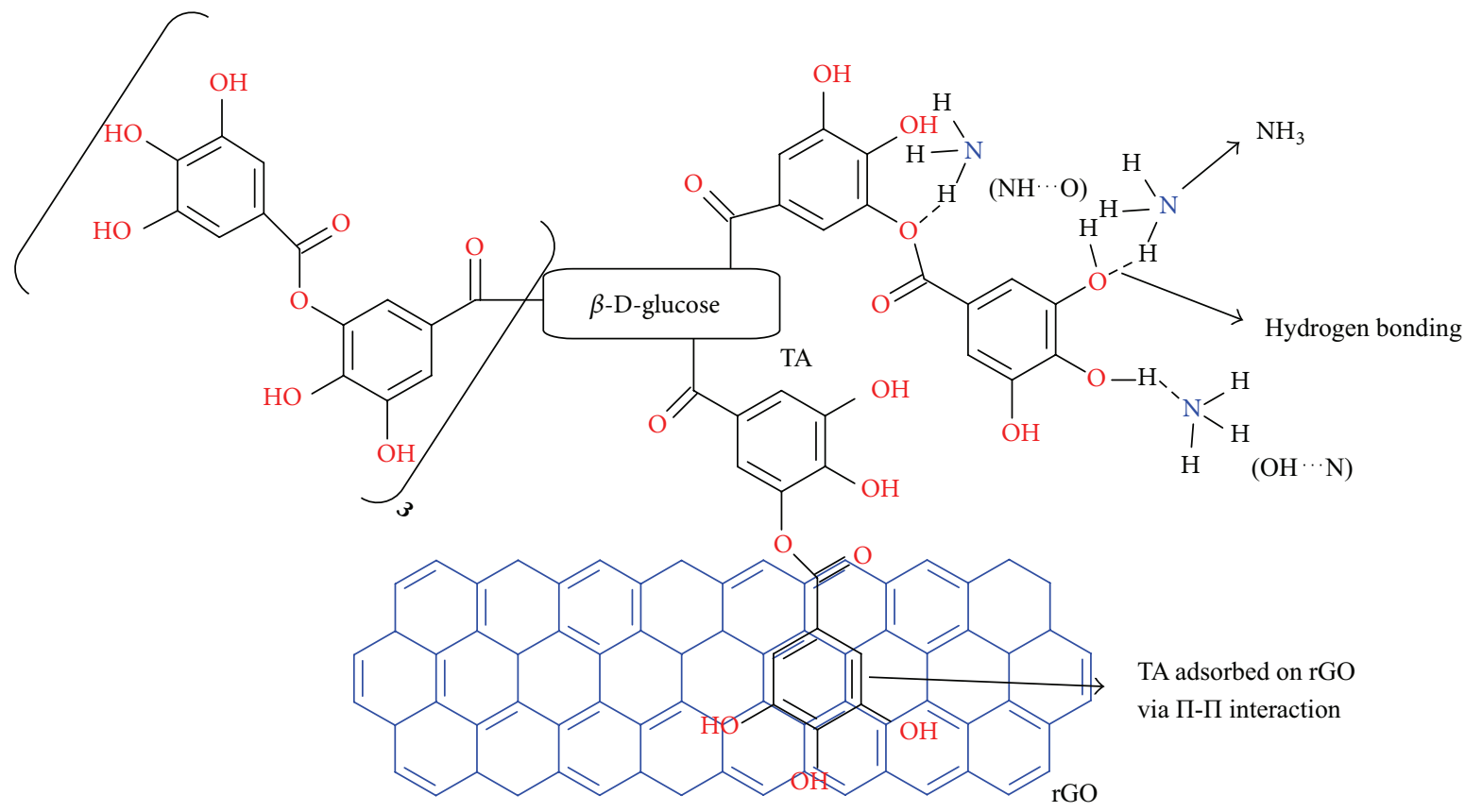

FIGURE 5: Illustration diagram of hydrogen bonding between hydroxyl and $\mathrm{NH}_{3}(\mathrm{OH} \cdots \mathrm{N})$, hydroxyl and $\mathrm{NH}_{3}(\mathrm{NH} \cdots \mathrm{O})$, and epoxide and $\mathrm{NH}_{3}(\mathrm{NH} \cdots \mathrm{O})$.

nucleophilic reactions and followed by elimination [21, 22]. From Figure 3, UV-Vis spectra of GO have absorption peaks at $228 \mathrm{~nm}$ and around $304 \mathrm{~nm}$ (small shoulder peak) due to $\Pi-\Pi^{*}$ transition of aromatic $\mathrm{C}-\mathrm{C}$ and $\mathrm{n}-\Pi^{*}$ transition of $\mathrm{C}=\mathrm{O}$ [2]. Upon reduction, the absorption peaks disappeared and new peak is found around $270 \mathrm{~nm}$ which again suggested the restoration of electronic conjugation $[23,24]$. TA has absorption peaks around 213 and $276 \mathrm{~nm}$ which are assigned for $\Pi-\Pi^{*}$ aromatic units and $\mathrm{C}=\mathrm{O}$ groups, respectively. Thus the absorption peak of $\mathrm{rGO}_{\mathrm{TA}}$ functionalized around $213 \mathrm{~nm}$ clearly indicated the sorption of TA [25]. The recovery of hexagonal networks is allowing the TA to adsorb on rGO with aromatic rings binding to the surface carbon rings via $\Pi-\Pi$ interactions $[21,26]$.

3.2. Gas Sensing Characteristic of $r G O_{T A}$ functionalized. The exposure of $\mathrm{rGO}_{\mathrm{TA}}$ functionalized to ammonia gas $\left(\mathrm{NH}_{3}\right)$ responded by decrement in electrical resistance ratio $R / R_{0}$ (Figure 4(a)). $R$ and $R_{0}$ are electrical resistance values with and without ammonia gas, respectively. Electrical resistance respond ratio $r_{\mathrm{RES}}$, which is defined as ratio in percentage: $\left(R_{0}-R / R_{0}\right) \times 100 \%$, is inversely proportional to $\mathrm{NH}_{3}$ concentration in linear (Figure 4(c)). A plausible sensing mechanism is established. TA is known to be electron donating molecules. TA molecules sorption via $\Pi$ - $\Pi$ stacking $n$-doped the $\mathrm{rGO}$, which is intrinsically p-type. The interaction between $\mathrm{NH}_{3}$ with $\mathrm{rGO}_{\mathrm{TA}}$ functionalized would be expecting the donation of negative charge carrier to $\mathrm{rGO}_{\mathrm{TA}}$ functionalized from $\mathrm{NH}_{3}$, in which $\mathrm{NH}_{3}$ is acting as donor characteristic [27]. Thus, the charge carrier transfer greatly enhances the negative charge carrier density of $\mathrm{rGO}_{\mathrm{TA}}$ functionalized, leading to decrement in overall electrical resistance which agreed well with the experimental observation [28, 29].

The exposure of $\mathrm{rGO}_{\mathrm{TA}}$ functionalized to continuous cycles of $\mathrm{NH}_{3}$ has demonstrated good consistency and reversibility of $\mathrm{rGO}_{\mathrm{TA}}$ functionalized in $\mathrm{NH}_{3}$ gas detection (Figure 4(b)). As from Figure $4(\mathrm{~d}), \mathrm{rGO}_{\mathrm{TA}}$ functionalized gave negligible electrical resistance ratio reading of conductance increment when exposed to organic vapours such as ethanol $(\approx$ $\left.0.31 \% R / R_{0}\right)$ and acetone $\left(\approx 0.32 \% R / R_{0}\right)$ in compare to $\mathrm{NH}_{3}\left(\approx 12.5 \% R / R_{0}\right)$. It clearly suggests the strong selectivity of $\mathrm{rGO}_{\mathrm{TA}}$ functionalized towards $\mathrm{NH}_{3}$. The strong selectivity towards $\mathrm{NH}_{3}$ may due to $\mathrm{NH}_{3}$ strong reducing and donor behaviour among the introduced vapours, where we evaluated that $\mathrm{NH}_{3}$ injected a much higher amount of negative charge carrier into $\mathrm{rGO}_{\mathrm{TA}}$ functionalized compared to other introduced vapours. Therefore greatly enhancing the negative charge carrier density of $\mathrm{rGO}_{\mathrm{TA}}$ functionalized.

Analysis of curves in Figure 4 revealed that it is an immediate sharp response of decrement in resistance followed by gradual recovery and saturation upon exposure to $\mathrm{NH}_{3}$. The gradual recovery region arises from desorption of the weak molecular adsorption onto low energy binding sites such as $\mathrm{sp}^{2}$ bonded carbon. Whereas saturation region arises from molecular interaction with higher energy binding sites, in particular oxygen functional group that is introduced by TA and residual oxygen functional group from incomplete reduction, which is substantially stronger $[4,30,31]$. The molecular interaction of $\mathrm{NH}_{3}$ with $\mathrm{rGO}_{\text {TA functionalized }}$ occurred through hydrogen bonding with hydroxyl and epoxide groups (Figure 5). The hydrogen bonding between $\mathrm{NH}_{3}$ and hydroxyl group is either occurred by coordination of the $\mathrm{N}$ in $\mathrm{NH}_{3}$ to the $\mathrm{H}$ in hydroxyl group $(\mathrm{OH} \cdots \mathrm{N})$ or 
TABLE 1: Respond ratio $/ r_{\mathrm{RES}}$, respond time $/ t_{\mathrm{RES}}$, recovery ratio $/ r_{\mathrm{REC}}$, and recovery time $/ t_{\mathrm{REC}}$ of $\mathrm{rGO}_{\mathrm{TA} \text { functionalized }}$ towards different concentration of $\mathrm{NH}_{3} / \mathrm{C}_{\mathrm{NH}_{3}}$.

\begin{tabular}{lcccc}
\hline $\mathrm{C}_{\mathrm{NH}_{3}}(\mathrm{ppm})$ & $r_{\mathrm{RES}}$ & $t_{\mathrm{RES}}(\mathrm{s})$ & $r_{\mathrm{REC}}$ & $t_{\mathrm{REC}}(\mathrm{s})$ \\
\hline 1310 & $9.3 \%$ & 40 & $66.45 \%$ & 170 \\
2620 & $12.5 \%$ & 40 & $88.87 \%$ & 90 \\
3930 & $14.9 \%$ & 40 & $93.80 \%$ & 250 \\
5240 & $17.8 \%$ & 20 & $100.00 \%$ & 260 \\
6550 & $20.1 \%$ & 20 & $100.00 \%$ & 100 \\
\hline
\end{tabular}

coordination of one of the $\mathrm{H}$ atoms in $\mathrm{NH}_{3}$ to the $\mathrm{O}$ in hydroxyl group $(\mathrm{NH} \cdots \mathrm{O})$. Whereas hydrogen bonding of $\mathrm{NH}_{3}$ with epoxide group is coordination of one of the $\mathrm{H}$ atoms in $\mathrm{NH}_{3}$ to the $\mathrm{O}$ of epoxide group $(\mathrm{NH} \cdots \mathrm{O})[32,33]$.

Strikingly, saturation region is recoverable quickly without the needs of stimulus in which recovery of molecular interaction is usually slow or unrecoverable [4]. This attributes to the good recovery ratio $r_{\mathrm{REC}}$ and fast recovery time $t_{\mathrm{REC}}$ (Table 1). $r_{\mathrm{REC}}$ is defined as ratio in percentage: $\left(\left(R-R_{a}\right) /\left(R-R_{0}\right)\right) \times 100 \% . R_{a}$ is electrical resistance values after ammonia gas desorption. $t_{\mathrm{REC}}$ is defined here as time required reaches $90 \%$ of initial resistance amplitude. It is to highlight that recovery ratio $r_{\mathrm{REC}}$ and recovery time $t_{\mathrm{REC}}$ of $\mathrm{rGO}_{\text {TA functionalized }}$ do not deteriorate with increases of $\mathrm{NH}_{3}$ concentration $\mathrm{C}_{\mathrm{NH}_{3}}$ even in regime of dense concentration (>3000 ppm).

The sorption of TA provides abundant amount of hydroxyl $(-\mathrm{OH})$ and epoxide $(=\mathrm{O})$ which serve as active site for gas absorption, thus enhancing the respond time and sensitivity [34]. The excellent respond times $t_{\mathrm{RES}}$ and recovery times $t_{\mathrm{REC}}$ ( $40 \mathrm{~s}$ and $260 \mathrm{~s}$, resp., for $1310-6550 \mathrm{ppm}$ of $\mathrm{NH}_{3}$ ) are assuring the good detection efficiency. Respond ratio $r_{\text {RES }}$ and recovery times are paramount in practical application. In addition, $\mathrm{rGO}_{\mathrm{TA}}$ functionalized has shown great improvement in dense concentration regime compared to previous studies, in particular on recovery ratio $r_{\mathrm{REC}}$, respond ratio $r_{\mathrm{RES}}$, and recovery times $t_{\mathrm{REC}}$ where $\mathrm{rGO}_{\mathrm{TA}}$ functionalized demonstrated respond time $t_{\mathrm{RES}}$ of $40 \mathrm{~s}$, recovery ratio of $93.8 \%$, and recovery time of $260 \mathrm{~s}[31,35]$.

\section{Conclusion}

In summary, we demonstrated a green yet simple $\mathrm{NH}_{3}$ gas sensor based on $\mathrm{rGO}_{\mathrm{TA}}$ functionalized . Electrical resistance decreases upon exposure to $\mathrm{NH}_{3}$ where we postulated that it is due to $\mathrm{n}$-doping by TA and charge transfer between $\mathrm{rGO}_{\mathrm{TA}}$ functionalized and $\mathrm{NH}_{3}$ through hydrogen bonding. Experimental findings revealed that $\mathrm{rGO}_{\mathrm{TA}}$ functionalized has high potential value to be explored for detection of $\mathrm{NH}_{3}$ based on excellent selectivity, respond times, and recovery times, especially under dense concentration regime ( $>3000 \mathrm{ppm})$. The stimulus-free, wide range detection capability and room temperature operation do favour the practical use of $\mathrm{rGO}_{\mathrm{TA} \text { functionalized }}$ in $\mathrm{NH}_{3}$ gas sensing.

\section{Conflict of Interests}

The authors declare that there is no conflict of interests regarding the publication of this paper.

\section{Acknowledgments}

This work was financially supported by the National Natural Science Foundation of China (Grant nos. 91123018, 61172041, 61172040, 50975226, and 60801022), the National 863 Key Project of Panel Displays (Grant no. 2008AA03A314), and the Fundamental Research Funds for the Central Universities.

\section{References}

[1] B. Timmer, W. Olthuis, and A. Van Den Berg, "Ammonia sensors and their applications-a review," Sensors and Actuators B: Chemical, vol. 107, no. 2, pp. 666-677, 2005.

[2] S. Pandey, G. K. Goswami, and K. K. Nanda, "Nanocomposite based flexible ultrasensitive resistive gas sensor for chemical reactions studies," Scientific Reports, vol. 3, p. 2082, 2013.

[3] J. D. Fowler, M. J. Allen, V. C. Tung, Y. Yang, R. B. Kaner, and B. H. Weiller, "Practical chemical sensors from chemically derived graphene," ACS Nano, vol. 3, no. 2, pp. 301-306, 2009.

[4] J. T. Robinson, F. K. Perkins, E. S. Snow, Z. Wei, and P. E. Sheehan, "Reduced graphene oxide molecular sensors," Nano Letters, vol. 8, no. 10, pp. 3137-3140, 2008.

[5] G. Lu, L. E. Ocola, and J. Chen, "Gas detection using lowtemperature reduced graphene oxide sheets," Applied Physics Letters, vol. 94, no. 8, Article ID 083111, 2009.

[6] S. Basu and P. Bhattacharyya, "Recent developments on graphene and graphene oxide based solid state gas sensors," Sensors and Actuators B: Chemical, vol. 173, pp. 1-21, 2012.

[7] X. Liu, S. Cheng, H. Liu, S. Hu, D. Zhang, and H. Ning, "A survey on gas sensing technology," Sensors, vol. 12, no. 7, pp. 9635-9665, 2012.

[8] A. Lipatov, A. Varezhnikov, P. Wilson, V. Sysoev, A. Kolmakov, and A. Sinitskii, "Highly selective gas sensor arrays based on thermally reduced graphene oxide," Nanoscale, vol. 5, no. 12, pp. 5426-5434, 2013.

[9] X. Huang, N. Hu, R. Gao et al., "Reduced graphene oxidepolyaniline hybrid: preparation, characterization and its applications for ammonia gas sensing," Journal of Materials Chemistry, vol. 22, no. 42, pp. 22488-22495, 2012.

[10] Y. Wang, Z. Shi, and J. Yin, "Facile synthesis of soluble graphene via a green reduction of graphene oxide in tea solution and its biocomposites," ACS Applied Materials \& Interfaces, vol. 3, no. 4, pp. 1127-1133, 2011.

[11] J. I. Paredes, S. Villar-Rodil, M. J. Fernández-Merino, L. Guardia, A. Martínez-Alonso, and J. M. D. Tascón, "Environmentally friendly approaches toward the mass production of processable graphene from graphite oxide," Journal of Materials Chemistry, vol. 21, no. 2, pp. 298-306, 2011.

[12] M. J. Fernández-Merino, L. Guardia, J. I. Paredes et al., "Vitamin $\mathrm{C}$ is an ideal substitute for hydrazine in the reduction of graphene oxide suspensions," Journal of Physical Chemistry C, vol. 114, no. 14, pp. 6426-6432, 2010.

[13] C. Zhu, S. Guo, Y. Fang, and S. Dong, "Reducing sugar: new functional molecules for the green synthesis of graphene nanosheets," ACS Nano, vol. 4, no. 4, pp. 2429-2437, 2010. 
[14] Y. Zhou, Q. Bao, L. A. L. Tang, Y. Zhong, and K. P. Loh, "Hydrothermal dehydration for the "green" reduction of exfoliated graphene oxide to graphene and demonstration of tunable optical limiting properties," Chemistry of Materials, vol. 21, no. 13, pp. 2950-2956, 2009.

[15] I. Mueller-Harvey, "Analysis of hydrolysable tannins," Animal Feed Science and Technology, vol. 91, no. 1-2, pp. 3-20, 2001.

[16] A. E. Hagerman, Hydrolyzable Tannin Structural Chemistry. Tannin Handbook, 2002, http://www.users.muohio.edu/hagermae/tannin.pdf.

[17] S. K. Sivaraman, I. Elango, S. Kumar, and V. Santhanam, "A green protocol for room temperature synthesis of silver nanoparticles in seconds," Current Science, vol. 97, no. 7, pp. 1055-1059, 2009.

[18] X. Tian, J. Li, and S. Pan, "Facile synthesis of single-crystal silver nanowires through a tannin-reduction process," Journal of Nanoparticle Research, vol. 11, no. 7, pp. 1839-1844, 2009.

[19] Y. Zhang, S. Liu, L. Wang et al., "One-pot green synthesis of Ag nanoparticles-graphene nanocomposites and their applications in SERS, H2O2, and glucose sensing," RSC Advances, vol. 2, no. 2, pp. 538-545, 2012.

[20] Y. Zhang, S. Liu, W. Lu, L. Wang, J. Tian, and X. Sun, "In situ green synthesis of Au nanostructures on graphene oxide and their application for catalytic reduction of 4-nitrophenol," Catalysis Science and Technology, vol. 1, no. 7, pp. 1142-1144, 2011.

[21] Y. Lei, Z. Tang, R. Liao, and B. Guo, "Hydrolysable tannin as environmentally friendly reducer and stabilizer for graphene oxide," Green Chemistry, vol. 13, no. 7, pp. 1655-1658, 2011.

[22] R. Liao, Z. Tang, Y. Lei, and B. Guo, "Polyphenol-reduced graphene oxide: mechanism and derivatization," Journal of Physical Chemistry C, vol. 115, no. 42, pp. 20740-20746, 2011.

[23] D. Li, M. B. Müller, S. Gilje, R. B. Kaner, and G. G. Wallace, "Processable aqueous dispersions of graphene nanosheets," Nature Nanotechnology, vol. 3, no. 2, pp. 101-105, 2008.

[24] M. Zainy, S. Vijay Kumar, H. N. Lim, C. H. Chia, and I. Harrison, "Simple and scalable preparation of reduced graphene oxidesilver nanocomposites via rapid thermal treatment," Materials Letters, vol. 89, pp. 180-183, 2012.

[25] M. G. Albu, M. V. Ghica, M. Giurginca, V. Trandafir, L. Popa, and C. Cotrut, "Spectral characteristics and antioxidant properties of tannic acid immobilized on collagen drug-delivery systems," Revista de Chimie, vol. 60, no. 7, pp. 666-672, 2009.

[26] D. Lin and B. Xing, "Tannic acid adsorption and its role for stabilizing carbon nanotube suspensions," Environmental Science and Technology, vol. 42, no. 16, pp. 5917-5923, 2008.

[27] H. Chang, J. D. Lee, S. M. Lee, and Y. H. Lee, "Adsorption of $\mathrm{NH}_{3}$ and $\mathrm{NO}_{2}$ molecules on carbon nanotubes," Applied Physics Letters, vol. 79, no. 23, pp. 3863-3865, 2001.

[28] B. Kumar, K. Min, M. Bashirzadeh et al., "The role of external defects in chemical sensing of graphene field-effect transistors," Nano Letters, vol. 13, no. 5, pp. 962-1968, 2013.

[29] G. Lu, L. E. Ocola, and J. Chen, "Gas detection using lowtemperature reduced graphene oxide sheets," Applied Physics Letters, vol. 94, no. 8, Article ID 083111, 3 pages, 2009.

[30] Y. Hajati, T. Blom, S. H. Jafri et al., "Improved gas sensing activity in structurally defected bilayer graphene," Nanotechnology, vol. 23, no. 50, Article ID 505501, 2012.

[31] S. Cui, S. Mao, Z. Wen, J. Chang, Y. Zhang, and J. Chen, "Controllable synthesis of silver nanoparticle-decorated reduced graphene oxide hybrids for ammonia detection," Analyst, vol. 138, no. 10, pp. 2877-2882, 2013.
[32] E. C. Mattson, K. Pande, M. Unger et al., "Exploring adsorption andreactivity of $\mathrm{NH}_{3}$ on reduced graphene oxide," The Journal of Physical Chemistry C, vol. 117, no. 20, pp. 10698-10707, 2013.

[33] J. A. Robinson, E. S. Snow, Ş. C. Bădescu, T. L. Reinecke, and F. K. Perkins, "Role of defects in single-walled carbon nanotube chemical sensors," Nano Letters, vol. 6, no. 8, pp. 1747-1751, 2006.

[34] K. Kim, H. Kang, C. Y. Lee, and W. S. Yun, "Enhanced response to molecular adsorption of structurally defective graphene," Journal of Vacuum Science \& Technology B, vol. 31, no. 3, Article ID 030602, 2013.

[35] R. Ghosh, A. Midya, S. Santra, S. K. Ray, and P. K. Guha, "Chemically reduced graphene oxide for ammonia detection at room temperature," ACS Applied Materials \& Interfaces, vol. 5, no. 15 , pp. 7599-7603, 2013. 

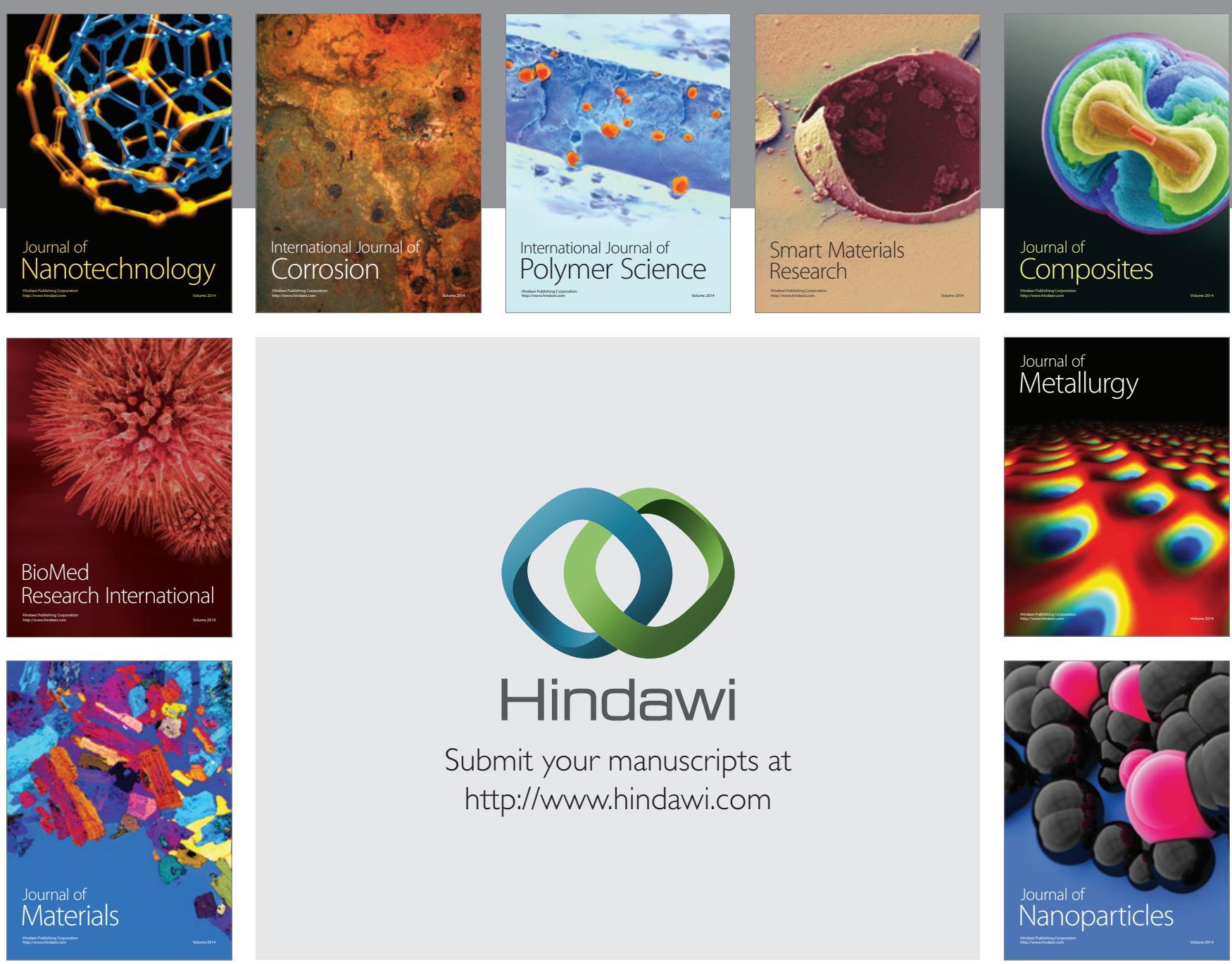

Submit your manuscripts at http://www.hindawi.com
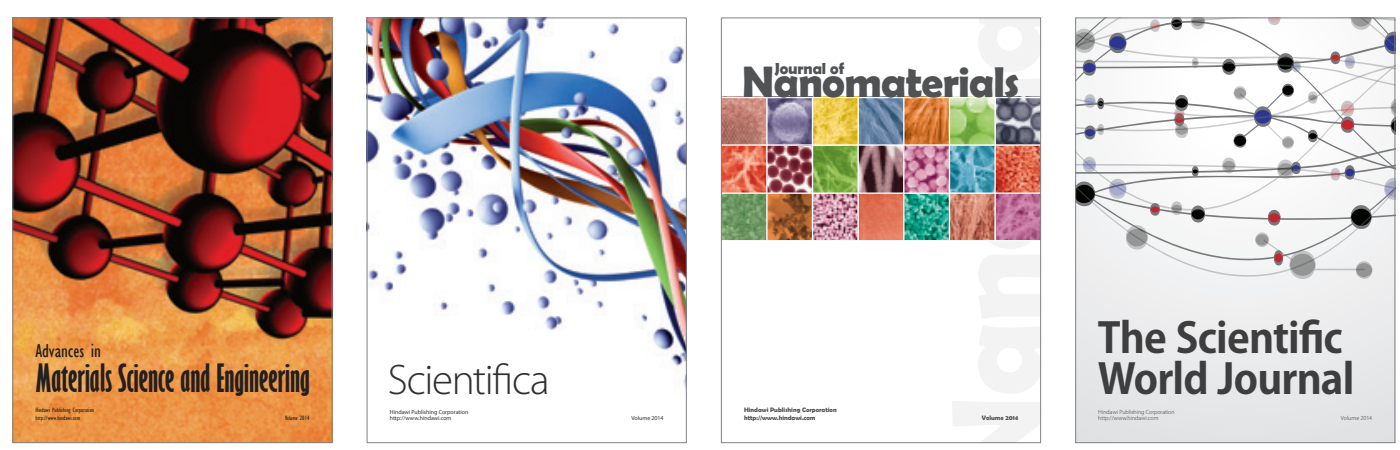

\section{The Scientific World Journal}
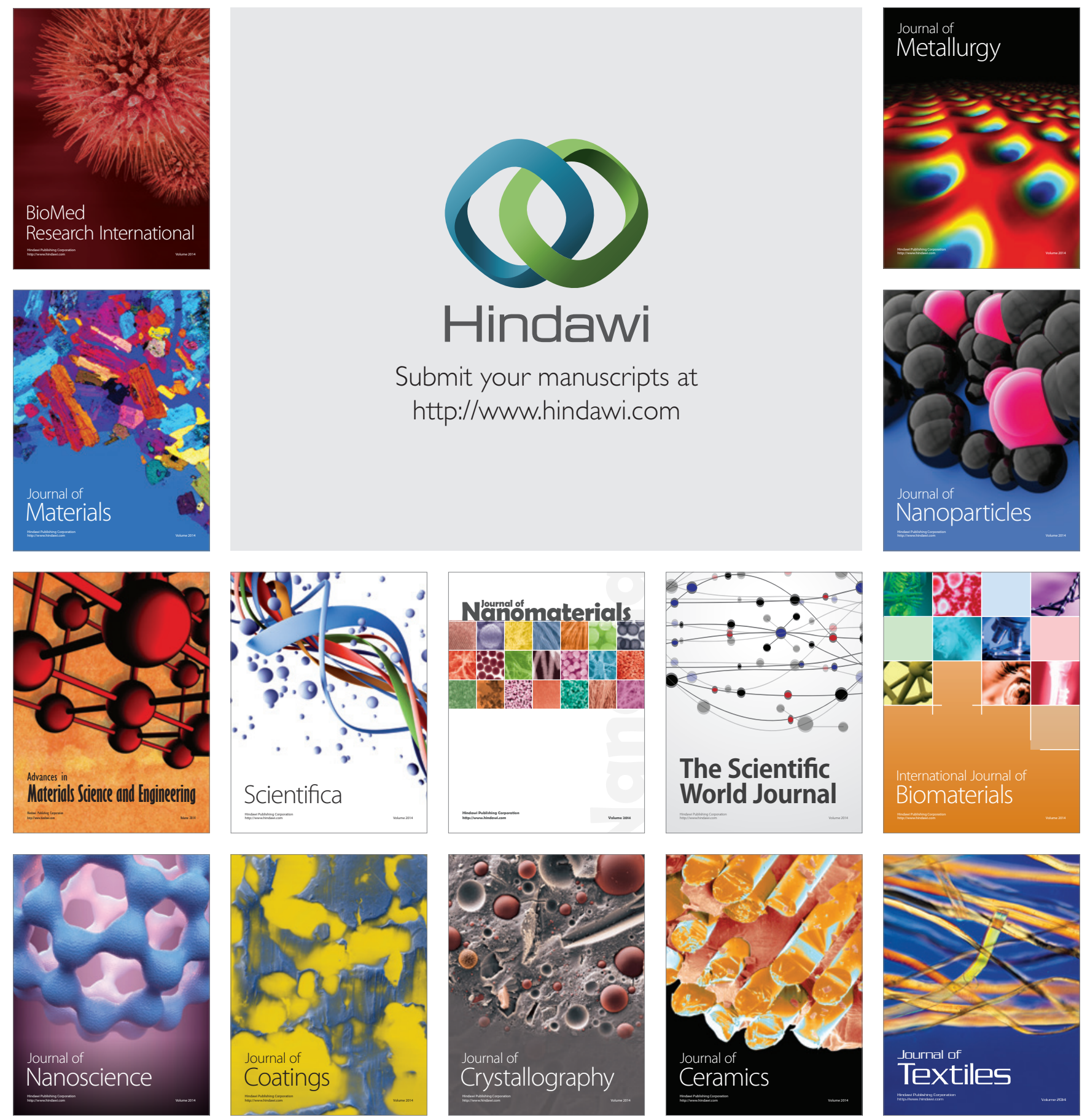